NASA CONTRACTOR REPORT

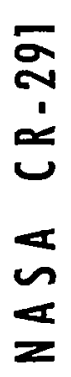

\title{
VIBRATIONAL ENERGY TRANSFER IN HIGH ENERGY COLLISIONS
}

by Charles E. Treanor

Prepared under Contract No. NASr-119 by

CORNELL AERONAUTICAL LABORATORY, INC.

Buffalo, N.Y.

for

NATIONAL AERONAUTICS AND SPACE ADMINISTRATION - WASHINGTON, D. C. - AUgUST 1965 


\section{VIBRATIONAL ENERGY TRANSFER IN HIGH ENERGY COLLISIONS}

By Charles E. Treanor

Distribution of this report is provided in the interest of information exchange. Responsibility for the contents resides in the author or organization that prepared it.

Prepared under Contract No. NASr -119 by CORNELL AERONAUTICAL LABORATORY, INC.

Buffalo, N.Y.

for

NATIONAL AERONAUTICS AND SPACE ADMINISTRATION

For sole by the Cleoringhouse for Federal Scientific and Technical Information Springfield, Virginia 22151 - Price $\$ 1.00$ 


\begin{abstract}
Time-dependent wave functions are used to evaluate the exact transition probabilities for a forced harmonic oscillator. The forcing is represented by a time-dependent potential, where this potential has a linear dependence on the oscillator coordinate. The results are compared with available numerical solutions for a harmonic oscillator forced with a potential which has an exponential dependence on the oscillator coordinate. The comparison is made for the collision of an $\mathrm{N}_{2}$ molecule with another particle, and it is found that although the results for the two cases are similar, the linear potential gives higher values for the multiquantum transitions. It is then shown that time dependent wave functions which contain the corresponding classical motion as a parameter provide a good set of functions for a perturbation calculation. The energy transfer to these oscillating wave functions is always identical to the energy transfer to the classical oscillator. Thus the perturbation value of the energy transfer represents the difference between the classical and the quantum mechanical result. It is shown that this value, which is zero for the linear forcing potential, is very small for higher order potentials, even at high velocity of impact. This demonstrates that classical calculations can be used to obtain the energy transfer in molecular collisions at high temperature.
\end{abstract}




\section{INTRODUCTION}

The relaxation of the vibrational degree of freedom in diatomic molecules has been a subject of interest in those gasdynamic flows where the temperature of the gas is such that the average molecular energy is of the order of the energy of a single vibrational quantum. Under these conditions the vibrational mode absorbs an appreciable fraction of the energy that is associated with the molecular species. At still higher temperatures, where the molecules dissociate, the vibrational relaxation is of interest as an intermediate step in the dissociation process. For both of these cases it is known that perturbation calculations of the transition probabilities ${ }^{l}$ involve assumptions that are violated in the important high energy collisions. Thus a reliable procedure has not been available for the extension of experimental vibrational relaxation data to the high temperature region. In the present paper it is shown that the difference between the classical and quantum mechanical solutions can be calculated directly, and this difference is small even for high energy collisions.

The magnitude of the error associated with first-order perturbation calculation has recently been demonstrated by Rapp and Sharp. ${ }^{2}$ They have performed numerical calculations of transition probabilities for strong interaction of a high speed incident particle with a nitrogen molecule, and have shown that their results are much different from perturbation calculations, even at temperatures as low as $5000^{\circ} \mathrm{K}$. An alternate procedure is the calculation of vibrational energy transfer on the basis of a purely classical model of the oscillator. ${ }^{3,4,5}$ It has frequently been pointed out ${ }^{6}$ that such classical calculations yield results for the energy transfer which agree with the 
quantum mechanical perturbation results. It has further been shown 7,8 that for the forced simple harmonic oscillator a much closer relation exists between the classical and quantum mechanical solutions, since the rate of absorption of vibrational energy is identical in the two cases. This result is obtained by Kerner ${ }^{7}$ by using time-dependent oscillating wave functions for the quantum mechanical solution and by Bartlett and Moyal ${ }^{8}$ by using momentum wave functions.

There remains the question, however, of the degree of accuracy that can be expected in using the classical solution in the problem where the oscillator is not driven by a purely time-dependent force. In this case the oscillatory wave functions no longer give an exact solution to the quantum mechanical problem, but do give a solution whose energy is identical to that of the classical problem. In the present paper this question is investigated by using the oscillatory wave functions as the set of functions in which to expand the solution for a perturbation calculation, and the magnitude of the perturbation is then a measure of the disagreement between the classical and the correct quantum mechanical calculations of the energy. The equations are evaluated for $\mathrm{N}_{2}$ collisions, and comparison is made with published numerical results for a 4 -level oscillator model. 2 The results show that the classical model should provide correct answers for the energy transfer to a simple harmonic oscillator for collision velocities many times greater than those that can properly be used in the usual first order perturbation treatment. 
FORCED HARMONIC OSCILLATOR

It is shown by Kerner in Ref. 7 that if a simple harmonic oscillator is forced by a force $F(t)$, the solution of the time dependent Schrodinger equation can be obtained in the form of an oscillating wave packet ${ }^{9}$ which does not spread with time. Thus if the Schrodinger equation for the oscillator is

$$
-\frac{\hbar^{2}}{2 M} \frac{\partial^{2} \psi}{\partial x^{2}}+\left[\frac{1}{2} k x^{2}-x F(t)\right] \psi=i \hbar \frac{\partial \psi}{\partial t}
$$

then the solution is given by

$$
\Psi_{m}(x, t)=N_{m} \exp \left\{\frac{\dot{i}}{\hbar}\left(M \dot{u} x-\int_{t_{0}}^{t}\left(\delta+E_{m}\right) d t\right)-\frac{1}{2} \alpha^{2}(x-u)^{2}\right\} H_{m}[\alpha(x-u)\}
$$

where, as for the unforced oscillator, $H_{m}$ is the $m$ th Hermite polynomial, $N_{m}^{2}=\alpha / \pi^{\frac{1}{2}} 2^{m} m !$ is a normalizing factor, and $\alpha^{2}=\frac{1}{\hbar} \sqrt{M k}$, with $M$ the reduced mass and $k$ the spring constant. The time $t_{0}$ is the time at which the forcing starts, $u$ is a function of the time, and $\delta=\frac{1}{2} M \dot{u}^{2}-\frac{1}{2} k u^{2}$. Thus the probability density $\psi^{*} \psi$ can be that of any stationary state of the oscillator, moving in toto with the motion $u(t)$. It is shown, ${ }^{7}$ then, that $u(t)$ is related to $F(t)$ by the ordinary differential equation

$$
M \ddot{u}+k u=F(t)
$$

Thus the center of the wave packet moves exactly as a classical harmonic

3 
oscillator would move if driven by the force $F(t)$. The expectation value of the energy of the oscillator is the energy that the classical oscillator would have, plus the energy associated with the quantum state. Thus

$$
\int \psi_{m}^{*} H \Psi_{m} d x=\frac{1}{2} M \dot{u}^{2}+\frac{1}{2} k u^{2}+\left(m+\frac{1}{2}\right) \hbar \omega
$$

The solution (Eq. (2)) represents a special case of a D'Alembert's principle for quantum mechanics. In general, if $\psi(x)$ is a solution for a potential $V(x)$, then $\phi(x, t)=\psi(x-u) \exp \left[\frac{\dot{i}}{\hbar}(x M \dot{u}-w(t))\right]$ is the solution for a potential

$$
U(x, t)=V(x-u)-x M \ddot{u}-\frac{1}{2} M \dot{u}^{2}+\dot{u}(t)
$$

where $u(t)$ and $w(t)$ are arbitrary functions of the time. Then if the potential $V(x)$ is moved with a motion $u(t)$ (resulting in a potential $V(x-u)$ ), and at the same time a second potential $-\chi M \ddot{u}$ is imposed, this second potential will just keep the wave packet centered on $V(x-2 x)$. Thus $-x M \ddot{u}$ is the "fictitious potential" which permits Newton's laws (or Schrbdinger's) to apply in the nonNewtonian system. The potential $V(x)=\frac{1}{2} k x^{2}$ is unique in that $U(x, t)$ can then be put in the form $U(x, t)=\frac{1}{2} k x^{2}-x F(t)$. In the same way the simple harmonic oscillator driven by a time-dependent force can be considered a unique problem in that the solution for the rate of change of internal energy is just equal to that which would be obtained from the classical problem. In the present paper we examine the question of how accurate such a classical analogy is in the case where the interaction potential is dependent on the oscillator coordinate in a non-linear fashion.

It is shown in Ref. 7 that, to display the transitions between stationary states of an oscillator, the wave function given by Eq. (2) can be expanded in 
terms of the harmonic oscillator functions

$$
\psi_{m}(x, t)=N_{m} \exp \left[-\frac{1}{2} \alpha^{2} x^{2}\right] H_{m}(\alpha x) \exp \left[-\frac{i}{2} E_{m} t\right]
$$

so that

$$
\psi_{m}(x, t)=\sum_{0}^{\infty} f_{m m}(t) w_{m}(x, t)
$$

The coefficients

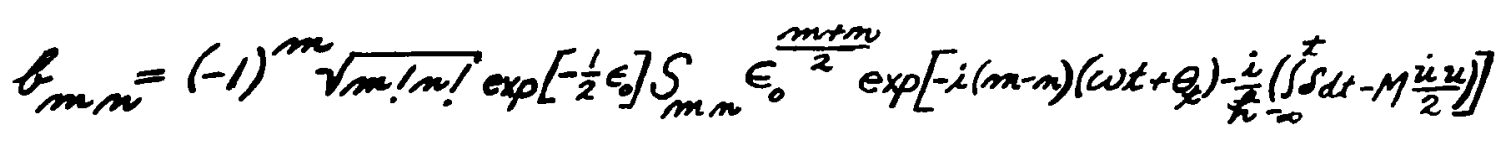

where

$$
S_{m m}=\sum_{j=0}^{\mu} \frac{(-1)^{j} \epsilon_{0}^{-j}}{(m-j) ! j !(m-j) !}
$$

and

$$
\theta_{t}=\tan ^{-1}\left[\frac{\dot{u}}{\omega u}\right]
$$

and $\mu$ is the lesser of $m, m$. The quantity $\epsilon_{0}$ is given by

$$
\epsilon_{0}(t)=\frac{\frac{1}{2} M \dot{u}^{2}(t)+\frac{1}{2} k u^{2}(t)}{\hbar \omega}
$$

Thus $\epsilon_{0}$ is equal to the energy that would have been absorbed by a classical oscillator driven by the force $F(t)$, divided by one quantum of energy for the true oscillator. Assuming that an oscillator starts in a stationary state m at $t=t_{1},($ ie. $u(t)=\dot{u}(t)=0$ ), and is then driven by a force $F(t)$, the proability that it is in the state $m$ at a time $t$ is given by ${ }^{7}$

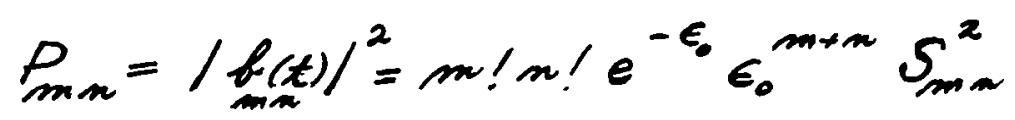

These relations permit multi-quantum transitions, as pointed out in Ref. 7 . Equation (10) is shown graphically in Fig. 1 for $m=0$ and several values of $m$. With $m=0$, Eq. $(10)$ is simply

5 


$$
P_{0 n}=\frac{e^{-\epsilon_{0}} \epsilon_{0}^{m}}{m !}
$$

Equation (10) is also obtained in Ref. 8, where the solution to the forced harmonic oscillator was obtained using momentum wave functions.

In the calculation of transition probabilities by perturbation methods, it is assumed that the energy transferred to the oscillator is small compared with a single quantum. This corresponds to $\epsilon_{0} \ll /$, and in this case $P_{m m}$ reduces to

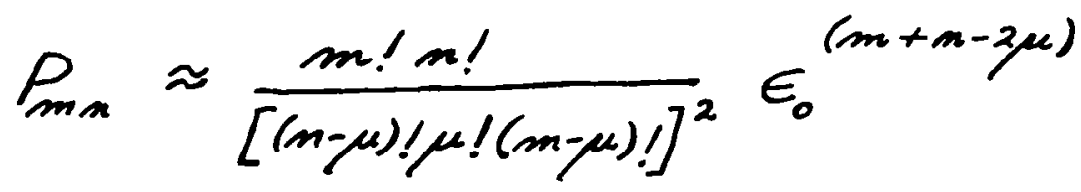

For $m=m-1$, there results

$$
P_{m, m-1} \approx m \epsilon_{0}
$$

which is the usual perturbation result for the simple harmonic oscillator ${ }^{1}$. Multi-quantum transitions depend on higher orders of $\epsilon_{0}$, and so their probabilities become equal to zero in perturbation treatment.

The success of the semi-classical approach to the harmonic oscillator problem is usually illustrated in terms of the perturbation result, wherein

$$
P_{0}=\epsilon_{0}
$$

Hence the rate of increase of vibrational energy is just equal to the rate that would be calculated classically. However, the oscillating wave packet results show that the connection between the quantum mechanical and the classical results is much more general, as illustrated in Eq. (4). This conclusion does not depend on a perturbation assumption, but only on the representation of the interaction potential in the form $\mathscr{X} F(t)$. In the case of collision of a particle with an oscillator, this representation implies that the interaction forces are 
long range compared both with the extent of the molecular wave function and with the amplitude of the oscillation of the coordinate $u(t)$. Departures from this condition certainly occur in high energy collisions, and are treated in the next section as a perturbation to the oscillating wave packet solution. The second assumption inherent in this approach is the representation of the effect of the colliding particle as a time-dependent potential. This approximation is discussed in Ref. 6. It requires that the vibrational energy gained by the oscillator be small compared with the total energy of the incident particle, a condition generally satisfied for collisions of interest in vibrational energy transfer. ${ }^{3}$ Unfortunately this condition is not satisfied in the case of an impulsive collision, for which an exact solution is available. 10 In this case the energy transferred to vibration is of the order of the initial translational energy, so the present results cannot be compared with those of Ref. 10 .

\section{APPLICATION TO MOLECULAR COLLISIONS}

In this section the vibrational transition probabilities calculated for a molecule which undergoes a high-speed collision with another particle are compared with those available from numerical calculations. ${ }^{2}$ To accomplish this the motion of the molecule and the colliding particle are uncoupled in an approximate way, using the method given by Rapp. ${ }^{2}, 4$ An incident particle $A$ collides with a molecule $B-C$, the initial relative velocity being $N_{0}$. The interaction is described by a repulsive potential $V(\mathfrak{A} X)$ between $A$ and $B$. (In Ref. 2, the notation $Y$ is used for the oscillator coordinate instead of $\mathcal{X}$.) It is a ssumed that the motion of the incident particle can be 
described classically and that it is sensibly independent of the degree of excitation of the molecule. The classical trajectory $X(t)$ of the incident particle is then inserted in the expression for the potential, so that $V(x, X)$ becomes $V(\psi, t)$, a function of the time and the oscillator coordinate.

There are two interaction potentials discussed in Ref. 2. The first is an exponential repulsion between $A$ and $B$,

$$
V(x, X)=E_{0} e^{-\frac{X}{L}} e^{\frac{m_{c}}{m_{c}+m_{c}} \frac{x}{L}}+\frac{1}{2} k x^{2}
$$

where

$$
E_{0}=\frac{1}{2} \tilde{n}_{0}^{2} \quad \tilde{m_{2}}=\frac{m_{1}\left(m_{0}+m_{c}\right)}{m_{1}+m_{0}+m_{0}}
$$

and $L$ is a parameter characterizing the range of the potential. The classical trajectory of particle $A$ in this potential, with $\mathscr{C}$ taken equal to zero, is given by

$$
e^{-\frac{X}{L}}=\operatorname{sech}^{2} \frac{v_{0} t}{2 L}
$$

An appropriate time-dependent potential can then be obtained for the oscillator by substitution of Eq. (14) into (13), giving

potential i $V_{I}(x, t)=E_{0} e^{\frac{x}{L}} \operatorname{sech}^{2}\left(\frac{n-t}{2 L}\right)+\frac{1}{2} k x^{2}$

where the substitution $L^{\prime}=L \frac{m_{b}+m_{c}}{m_{c}}$ has also been made.

A second potential, very similar to (15), is introduced in Ref, 2 in order to obtain an analytic solution to the problem of a two-level vibrator. We intraduce the second potential here because we wish to use it in the comparison of results with Ref. 2 . This potential is

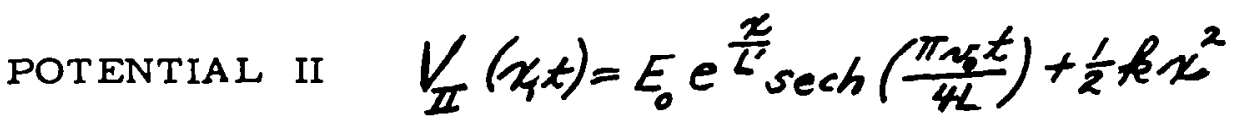

8 


\section{Linear Approximation}

The oscillator transition probabilities given in Eq. (10) are obtained for a collision of an incoming particle with the oscillator in the case where the potential can be described as $V(x, t)=\frac{1}{2}\left(x^{2}-x F(t)\right.$. If the range of the interacting force is large ( $K /{ }^{\prime}$ small), Eqs. (15) and (16) can be written in this form. Expanding potential II in powers of $K / \mathcal{L}^{\prime}$ and neglecting the purely time-depend ent terms in the potential then results in

$$
F_{I}=-\frac{E_{0}}{L^{\prime}} \operatorname{sech}\left(\frac{\pi r_{0} t}{4 L}\right)
$$

The classical energy transferred by this force is to be obtained in the usual way from Eq. (3). This energy supplies the numerator of Eq. (9) so that $\epsilon_{0}$ can be calculated. This solution is given for force I in Ref. 4; a similar treatment of force II produces

$$
\epsilon_{i I}=\frac{2}{\hbar \omega} \frac{\tilde{m}^{2}}{\mu}\left(\frac{m_{c}}{m_{0}+m_{0}}\right)^{2} \mu_{0}^{2} \operatorname{sech}^{2}\left(\frac{2 \omega L}{\mu_{0}}\right)
$$

The transition probabilities can be calculated by using Eq. (18) with Eq. (10) (or Fig. 1).

Following Ref. 2, we take, for $\mathrm{N}_{2}-\mathrm{N}_{2}$ collisions, $L=0.2 \AA$, $\omega=4.45 \times 10^{14} \mathrm{sec}^{-1}, m_{A}=2 m_{B}=2 m_{c}=4.65 \times 10^{-23} \mathrm{gms}$. The transition probabilities obtained in this way for potential II are shown in Fig. 2. The results for potential I are similar, except for low velocity collisions, where the details of the potential are very important. Also shown in Fig. 2 are the transition probabilities obtained numerically by Rapp and Sharp for a 4-level oscillator, using the potential Ir. It is seen that their values for $P_{O}$ agree with those obtained here, up to velocities of about $5 \times 10^{5} \mathrm{~cm} / \mathrm{sec}$. However, their values for higher order transitions are in considerable disagreement 
with the simple relation given by Eq. $\{11\}$, for $E_{0}<<1$

$$
P_{o m}=p_{01}^{n} \frac{1}{n !}
$$

This is illustrated in Fig. 3, where their values of $P_{a n}$ divided by $P m / m !$ are shown to be much different from unity, even for velocities as small as $2 \times 10^{5} \mathrm{~cm} / \mathrm{sec}$. The lack of agreement for high energy collisions would be expected, since Rapp and Sharp use the exponential potential of Eq. (16), where the present method uses only the leading term, as given in Eq. (17). The disagreement at lower velocities is surprising, however, since the maximum value of $\frac{\mu}{L}$ at $\varkappa_{0}=3 \times 10^{5} \mathrm{~cm} / \mathrm{sec}$ is $\sim 0.04$.

\section{Perturbation of Oscillatory Wave Functions}

If the potential in which the oscillator moves is not of the form indicated in Eq. (1), the oscillatory wave functions given in Eq. (2) will not provide solutions to the Schrodinger equation. However, these functions can be used as the set in which to expand the solution for the usual time-dependent perturbation method. Since the ground-state wave function oscillating with the classical motion contains the energy of a classical oscillator, the perturbation calculations which use such wave functions will supply a measure of the departure of the energy of the forced oscillator from the classically calculated value.

Consider a potential of the form

$$
V(x, t)=\frac{1}{2} \rho x^{2}-P(x, t)
$$

where $P(x, t)$ is the time-dependent potential driving the oscillator. The zero order potential is taken as 


$$
V_{0}(x, t)=\frac{1}{2} k x^{2}-x P^{\prime}(x, t)+U(t)
$$

which includes an arbitrary function of the time $J(t)$ to adjust the phase of the zero-order wave functions. The prime denotes differentiation with respect to $\mathcal{X}$. The wave functions then move centered at $u(t)$, where $u(t)$ follows the classical motion determined by

$$
m \ddot{u}+k u=P(u, t)
$$

Thus $P(u, t)$ replaces the force $F(t)$ in Eq. (3). The motion of the center of the wave packet, $\boldsymbol{u}(t)$, is then just equal to the motion of a classical oscillator acted on by the non-linear force.

It should be pointed out here that this classical problem, (Eq. 22), cannot be solved analytically, and so the calculation presented here does not lead to a closed-form answer. The purpose of the present calculation is rather to calculate the difference between the classical and quantum-mechanical results, that difference being represented by the perturbation. (An alternate procedure would be to consider the symmetric force $P^{\prime}(0, t)$ as the zeroorder term, so that the classical problem can be solved. The perturbation then represents the difference between the two results shown in Fig. 2. However, this calculation has not been given in the present paper.)

The perturbing potential is then

$$
V\left(x_{1}, t\right)-V_{0}(x, t)=V^{\prime}(z, t)=-\left[P(z+u, t)-(z+u) P^{\prime}(u, t)\right]-J(t)
$$

where the variable $\mathcal{Z}$ has been substituted for the quantity $\mathcal{X}-\boldsymbol{U}$, which appears as the argument in Eq. (2). For the case of a potential of the form of Eq. (16), $P(x, t)=-E_{0} e^{\frac{x}{L}}$ sech $\frac{\pi v_{0} t}{4 L}$ and Eq. (23) becomes 


$$
V^{\prime}(z, t)=-\frac{E_{0}}{L^{\prime}} e^{\frac{u}{L^{\prime}}} \operatorname{sech} \frac{\pi r_{0} t}{4 L}\left[L^{\prime} e^{\frac{z}{L^{\prime}}}-u-z\right]-J(t)
$$

Proceeding as usual for a perturbation calculation, the solution $\Psi(x, t)$ to the Schrodinger equation for $V(x, t)$ can be written in terms of the $\Psi_{m}(x, t)$ which are the solutions for the potential $V_{0}\left(x_{1}, t\right)$. Thus

$$
\Psi(x, t)=\sum_{m} a_{m j}(t) \Psi_{m}(x, t)
$$

where the functions $\psi_{\operatorname{m}}(x, t)$ are given in Eq. (2), and the subscript $j$ is used to identify the wave function $\psi_{j}\left(x_{3}-\infty\right)$ which describes the oscillator before the collision. Then

$$
i \hbar \dot{a}_{m j}=\sum_{m} a_{m j} V_{m m}^{\prime}(t)
$$

where

$$
V_{m m}^{\prime}(t)=\int_{-\infty}^{\infty} \psi_{m}^{*}(x, t) V^{\prime}(x, t) \Psi_{m}(x, t) d x
$$

and the integral is actually performed on the variable $\mathcal{Z}$ rather than $\mathbb{X}$. $J(t)$ is chosen to make $V_{j j}^{\prime}(t)=0$. To obtain the relations for transitions between stationary states, let the oscillator initially $(t=-\infty)$ be in a state $j$. Assume that $V(f, t)$, as given by $(20)$, satisfies the condition $V(x, \pm \infty)=\frac{1}{2} \not x_{x}^{2}$. Then

$$
\begin{array}{ll}
f_{m k}(-\infty)=\delta_{m k} & \Psi_{m}\left(x_{1}-\infty\right)=v_{m}\left(x_{j}-\infty\right) \\
a_{m j}(-\infty)=\delta_{m j} & \Psi\left(x_{1}-\infty\right)=v_{j}\left(x_{1},-\infty\right)
\end{array}
$$

and the probability that the oscillator is in a state $m$ at time $t=+\infty$ is

$$
\rho_{j m}=\left|\int_{-\infty}^{\infty} \Psi\left(x_{\infty} \infty\right) u_{m}^{*} d x\right|^{2}=\left|\sum_{m} a_{m j}(\infty) b_{m}(\infty)\right|^{2}
$$

12 
For first-order perturbation from the oscillating wave function, all $a_{\text {mg }}(t)$ in the sum are taken equal to their value at $t=-\infty$, viz, $a_{\text {mg }}(-\infty)=\delta_{\text {mg }}$. There results, then, the approximate expression

$$
a_{m j}(\infty)=\frac{1}{i=} \int_{-\infty}^{\infty} V_{m j}^{\prime} d t+\delta_{m j}
$$

Equations (30), (29) and (7) can then be combined so that the expression for the transition probability is obtained as

$$
P_{j \rightarrow n}=n ! e^{-\epsilon_{0}} \epsilon_{0} \sum_{m=0}^{\infty} \sum_{k_{0}}^{\infty}(-1)^{m+k} \sqrt{m / k !} \epsilon_{0}^{\frac{m+n}{2}} S_{m m} S_{k_{x}} a_{m j} \bar{a}_{k j} e^{-i(m-k) \phi}
$$

where $\phi$ is a phase factor given by the condition that the final classical motion is

$$
u=A \cos (\omega t-\phi)
$$

The transition probabilities given in Eq. (10) for the unperturbed case are inclued in this sum as the term with $\ell=m=j$, while the other terms represent the departure from the oscillation of a single wave function. For a force that is symmetric in $t, \phi$ is equal to $\pi / 2$. Some improvement can be made in the form of Eq. (31) by combining the terms with $m$ and $f$ interchanged, so that

$$
\begin{aligned}
& p_{j \rightarrow m}=n ! e^{-\epsilon_{0}} \epsilon_{0}^{n} \int \sum_{m=0}^{\infty} m / \epsilon_{0}^{m} S_{m m}^{2}\left(\delta_{m j}+I_{m j}^{2}\right)
\end{aligned}
$$

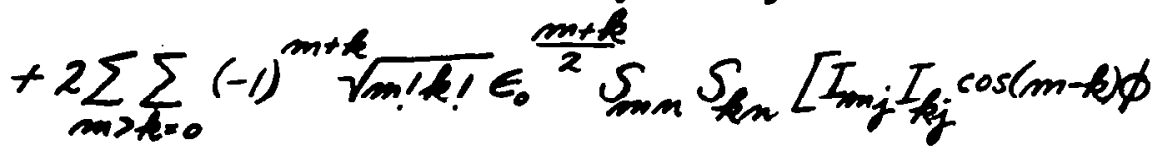

$$
\begin{aligned}
& \left.\left.f\left(\delta_{\operatorname{mij}} I_{\beta_{j}}-\alpha_{j} T_{\operatorname{mij}}\right) \sin \left(\operatorname{mon}-x_{k}\right) \phi\right]\right\}
\end{aligned}
$$

where

$$
I_{m j}=\frac{1}{\hbar} \int_{-\infty}^{\infty} V_{m j}^{\prime} d t
$$

13 
and $J(t)$ has been chosen so that $I_{j i}=0$.

To obtain a specific numerical result, consider the expansion of the potential given in Eq. $(16)$ in powers of $K / L^{\prime}$, and retain terms through $\left.(K /)^{\prime}\right)^{2}$. Then, from Eq. (20)

$$
P(x, t)=-E_{0} \operatorname{sech} \frac{\pi 2 \pi t}{4 L}\left[1+\frac{x}{L^{\prime}}+\frac{L}{2}\left(\frac{1}{L^{\prime}}\right)^{2}\right] ; P^{\prime}(x, t)=-\frac{E_{0}}{L^{\prime}} \operatorname{sech} \frac{\pi r_{0} t}{4 L}\left(1+\frac{x}{L^{\prime}}\right)
$$

So that Eq. (23) gives

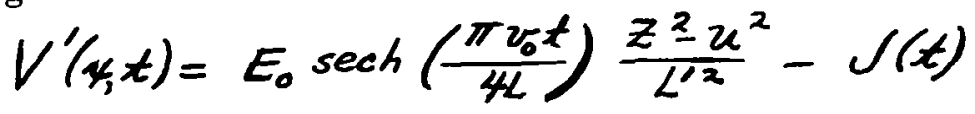

where $u$ is defined by

$$
m \ddot{u}+k_{u}=-\frac{E_{0}}{L^{\prime}} \operatorname{sech}\left(\frac{\pi u_{0} t}{4 L}\right)\left(1+\frac{u}{L^{\prime}}\right)
$$

From Eq. (27) and Ref.9,

$$
\begin{aligned}
& V_{\text {min }}^{\prime}(t)=-\frac{P(0, t)}{2 L^{\prime 2}}\left\langle z^{2}\right\rangle_{\text {mon }} e^{\frac{i}{x^{\prime}}\left(E_{m}-E\right) t}+\left(\frac{u^{2}}{2 L^{\prime 2}} P(0, t)-J(t)\right) \delta_{\text {m m }}
\end{aligned}
$$

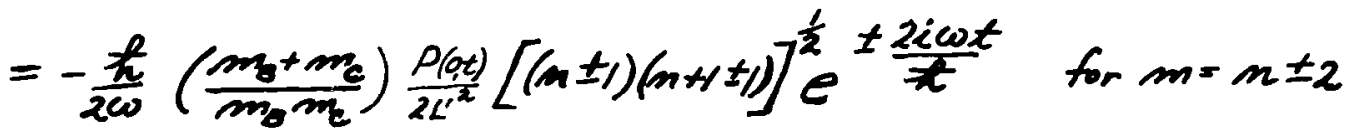

$$
\begin{aligned}
& =-\frac{\hbar L}{2 \omega}\left(\frac{m_{B}+m_{c}}{m_{0} m_{c}}\right) \frac{P(0, t)}{2 L^{\prime 2}}(2 n+1)+\left(\frac{u^{2}}{2 L^{2}} P(0, t)-V(t)\right) \quad \text { for } m=m \\
& =0 \text { otherwise, }
\end{aligned}
$$

and from (32)

$$
I_{j \pm 2, j}=-\frac{m_{A} m_{0}}{2 m_{0}\left(m_{1}+m_{0}+m_{0}\right)} \frac{v_{e}}{L \omega}[(j \pm 1)(j+1 \pm 1)]^{\frac{1}{2}} \operatorname{sech} \frac{4 \omega L}{v_{0}}
$$

and $T_{j j}=0$. Thus for $j=0$

$$
P_{0 \rightarrow m}=m ! e^{-\epsilon_{0}} \epsilon_{0}^{n}\left\{S_{0 m}^{2}+2 ! \epsilon_{0}^{2} S_{2 m}^{2} I_{20}^{2}-2 \sqrt{2} \epsilon_{0} S_{2 m} S_{0 m} I_{20} \sin 2 \phi\right\}
$$

Using the figures given before for $\mathrm{N}_{2}-\mathrm{N}_{2}$ collisions, and Eq. (8) for $S_{0 m}, S_{2 m}$

14 


$$
\begin{aligned}
& I_{20}=\frac{\sqrt{2} v_{0}}{3.56 \times 10^{6}} \operatorname{sech}\left(\frac{3.56 \times 10^{6}}{v_{0}}\right) ; \\
& S_{0 n}=\frac{1}{n !} ; \\
& S_{2 n}=\frac{1}{2}-\frac{\left(1-\delta_{0 n}\right)}{(m-1) ! \epsilon_{0}}+\frac{\left(1-\delta_{0 m}\right)\left(1-\delta_{1 n}\right)}{2(m-2) ! \epsilon_{0}^{2}}
\end{aligned}
$$

where $V_{0}$ is given in $\mathrm{cm} / \mathrm{sec}$. The oscillatory wave function result (and therefore, for the energy, the classical result) is given by the first term. Thus the departure from the classical result for the rate of energy absorption is

$$
\begin{aligned}
\Delta E & =E_{Q M}-E_{c}=\sum_{m=0}^{\infty} m \hbar \omega\left(P_{0 m}-m ! e^{-\epsilon_{0}} \epsilon_{0} m S_{0 m}^{2}\right) \\
& =\hbar \omega e^{-\epsilon_{0}}\left\{2 \epsilon_{0}^{2} I_{20}^{2} \sum_{0}^{\infty} m(m !) \epsilon_{0}^{m} S_{2 m}^{2}-2 \sqrt{2} \epsilon_{0} I_{20} \sin 2 \phi \sum_{0}^{\infty} m(m !) \epsilon_{0} S_{2 m} S_{0 m}\right\}
\end{aligned}
$$

Using Eq. (33) for $S_{2 n}$, the summations can be performed to give

$$
\Delta E=\hbar \omega T_{20}^{2}\left(2+E_{0}\right)
$$

Since the classical result is just $E_{c}=h \omega \epsilon_{0}$, the fractional departure is

$$
\frac{\Delta E}{E_{0}}=\frac{I_{20}^{2}}{2+\epsilon_{0}}
$$

This fraction is very small, even for large velocities; e.g. for $\psi_{0}=10^{6} \mathrm{~cm} / \mathrm{sec}$,

$$
\frac{\Delta E}{E_{c}}=5.1 \times 10^{-4}\left(\frac{2+\epsilon_{0}}{\epsilon_{0}}\right)
$$

Thus, even though the classical result for the energy transferred $\left(t \omega \epsilon_{0}\right)$ has not been evaluated, this equation shows that the classical answer will be very close to the quantum mechanical result. 


\section{SUMMARY}

It is shown that the linear forced-harmonic-oscillator solution of Kerner can be used to obtain an approximate solution for vibrational transitions of a diatomic molecule. The transition probabilities that are obtained are compared with the numerical solution of Rapp and Sharp for $\mathrm{N}_{2}-\mathrm{N}_{2}$ collisions. An oscillating forced-harmonic-oscillator solution is then used as the basis set for a perturbation calculation, where the magnitude of the energy transfer associated with the perturbation is equal to the difference between the correct quantum mechanical result and the classical result. It is shown that this value is small, even for high-energy collisions. Thus a classical calculation of the energy transfer between a simple harmonic oscillator and a collision partner should give an accurate result, and the small difference between it and the quantum mechanical result can be evaluated. 


\section{REFERENCES}

1. R. N. Schwartz, Z. I. Slawsky, and K. F. Herzfeld, J. Chem. Phys. 20, $1591(1952)$.

2. D. Rapp and T. E. Sharp, J. Chem. Phys. 38, 2641 (1963).

3. S. W. Benson, G. C. Berend, J. C. Wu, J. Chem. Phys. 38,25 (1963).

4. D. Rapp, J. Chem. Phys. 32, 735 (1960).

5. J. C. Parker, Phys, Fluids 2, 449 (1959).

6. D. Rapp, J. Chem. Phys. 40, 2813 (1964).

7. E. Kerner, Can. J. Phys. 36, 371 (1958).

8. M. S. Bartlett and J. E. Moyal, Proc. Cambridge Phil. Soc. 45, 545 (1949).

9. L. I. Schiff, Quantum Mechanics. McGraw-Hill Book Company, Inc. (1949).

10. K. E. Shuler and R. Zwanzig, J. Chem. Phys. 33, 1778 (1960). 


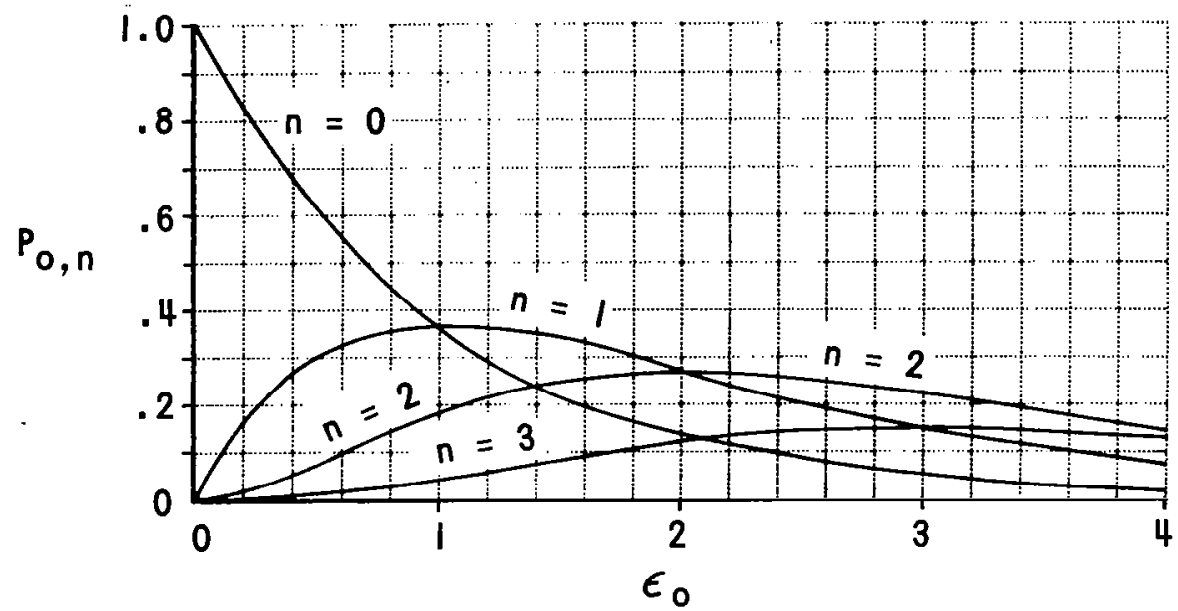

Figure I TRANSITION PROBABILITIES FOR HARMONIC OSCILLATOR WITH LINEAR FORCING AS FUNCTION OF CLASSICAL ENERGY TRANSFERRED, REF. 7

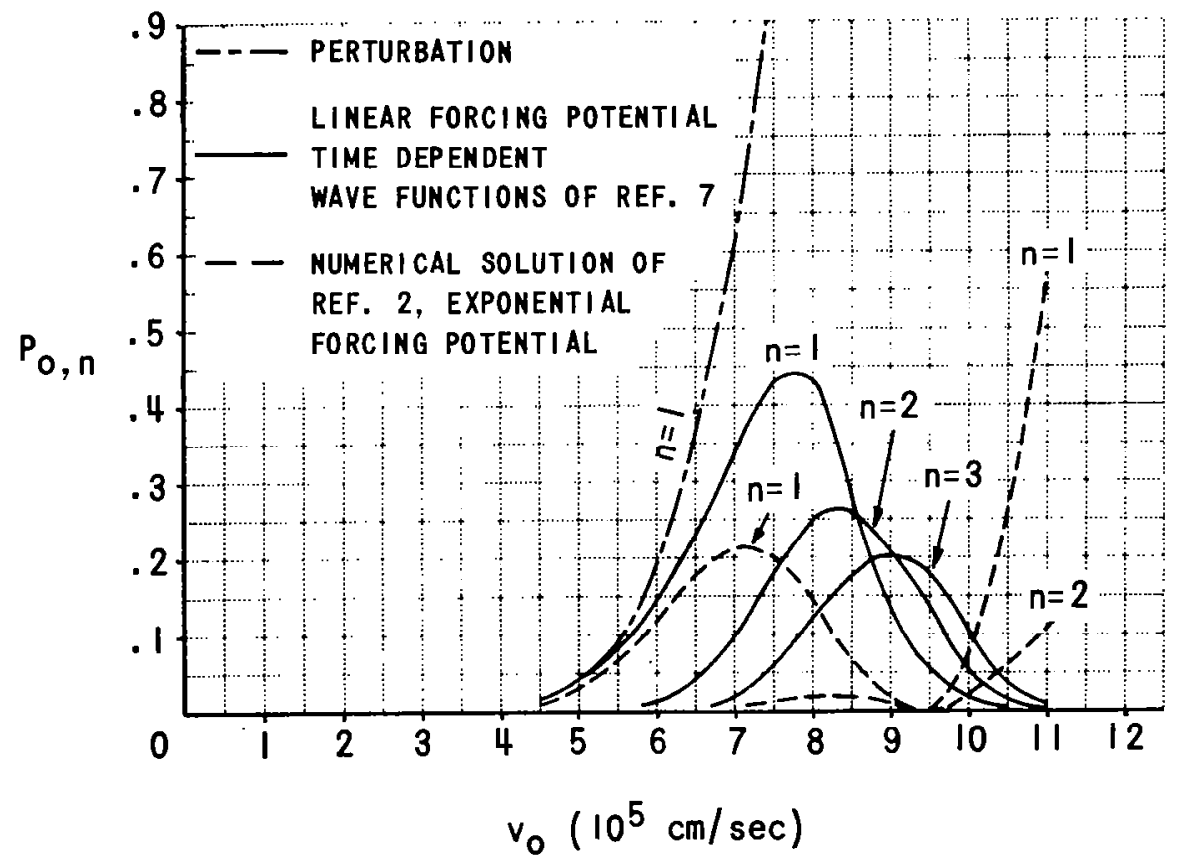

Figure 2 TRANSITION PROBABILITIES FOR $\mathrm{N}_{2}-\mathrm{N}_{2}$ COLLISIONS 


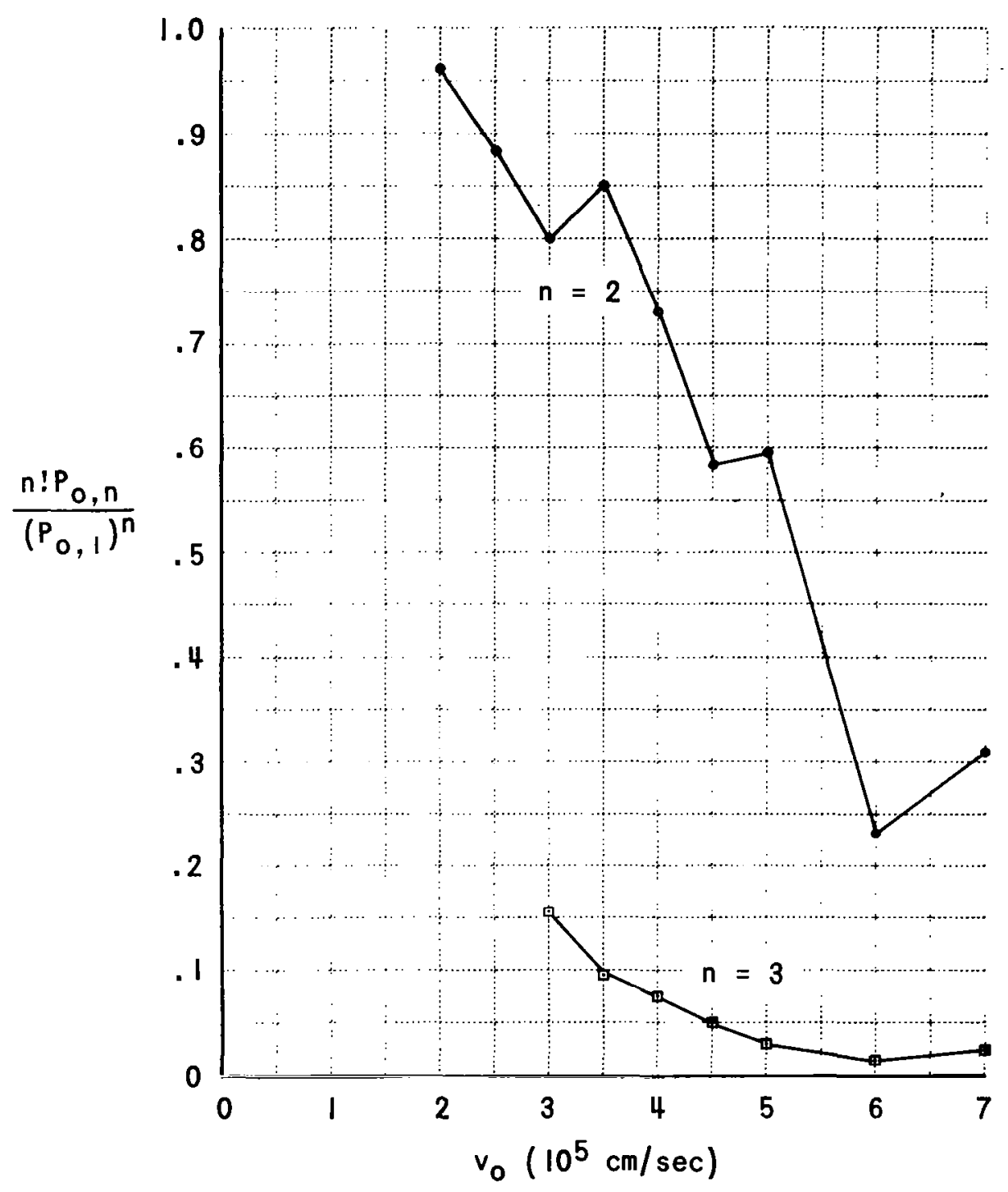

Figure 3 TRANSITION PROBABILITY RATIO, EQUATION 19, FOR EXPONENTIAL FORCING FUNCTION, REF. 2

$N_{2}-N_{2}$ COLLISIONS 
"The aeronautical and space activities of the United States shall be conducted so as to contribute. . . to the expansion of buman knowledge of phenomena in the atmospbere and space. The Administration shall provide for the widest practicable and appropriate dissemination of information concerning its attivities and the results thereof."

-National Aeronautics and Space Act of 1958

\section{NASA SCIENTIFIC AND TECHNICAL PUBLICATIONS}

TECHNICAL REPORTS: Scientific and technical information considered important, complete, and a lasting contribution to existing knowledge.

TECHNICAL NOTES: Information less broad in scope but nevertheless of importance as a contribution to existing knowledge.

TECHNICAL MEMORANDUMS: Information receiving limited distribution because of preliminary data, security classification, or other reasons.

CONTRACTOR REPORTS: Technical information generated in connection with a NASA contract or grant and released under NASA auspices.

TECHNICAL TRANSLATIONS: Information published in a foreign language considered to merit NASA distribution in English.

TECHNICAL REPRINTS: Information derived from NASA activities and initially published in the form of journal articles.

SPECIAL PUBLICATIONS: Information derived from or of value to NASA activities but not necessarily reporting the results of individual NASA-programmed scientific efforts. Publications include conference proceedings, monographs, data compilations, handbooks, sourcebooks, and special bibliographies.

Details on the availability of these publications may be obtained from:

SCIENTIFIC AND TECHNICAL INFORMATION DIVISION

NATIONAL AERONAUTICS AND SPACE ADMINISTRATION

Washingion, D.C. 20546 